\title{
Histological and Ultrastructural Features in the Early Stage of Purkinje Cell Degeneration in the Cerebellar Calcification (CC) Rat
}

\author{
Yosuke ANDO1), Nobutsune ICHIHARA2), Shigehito TAKESHITA ${ }^{1)}$, \\ Yoshiaki SAITO ${ }^{3)}$, Takeki KIKUCHI ${ }^{4}$, and Noboru WAKASUGI ${ }^{5)}$
}

\footnotetext{
${ }^{1)}$ Medicinal Safety Research Laboratories, Sankyo Co., Ltd., 717 Horikoshi, Fukuroi, Shizuoka 437-0065,

${ }^{2)}$ Department of Anatomy I, School of Veterinary Medicine, Azabu University, 1-17-71 Fuchinobe, Sagamihara, Kanagawa 229-8501, ${ }^{3}$ Hatano Research Institute, Food and Drug Safety Center, 729-5 Ochiai, Hadano, Kanagawa 257-8523, 4)Department of Animal Models for Human Disease, National Institute of Neuroscience, NCNP, 4-1-1 Ogawa-higashi-machi, Kodaira, Tokyo 187-8502, and ${ }^{5)}$ Laboratory of Animal Reproduction, Graduate School of Bioagricultural Sciences, Nagoya University, Furo-cho, Chikusa-ku, Nagoya 464-8601, Japan
}

\begin{abstract}
The cerebellar calcification (CC) rat is a new neurodegenerative mutant with severe Purkinje cell loss and symmetrical calcifications in the cerebellar cortex manifesting ataxia: lack of coordination in body movements. In the present study, histopathological features were examined in the Purkinje cell degeneration in postnatal homozygous suckling rats without clinical signs, which were genotyped by microsatellite markers. In addition, the calcified Purkinje cells were investigated ultrastructurally and elemental analysis was performed on the deposits. Body weight of the homozygous (cc/cc) rats was already slightly lower compared with the heterozygotes $(\mathrm{ccl}+)$ in the neonatal stage. The degeneration of the Purkinje cells in the cc/cc rats was recognized obviously in lobules VI, VII, VIII and IX from 14 days after birth, a few days before the appearance of the ataxic behavior. The Purkinje cells in the region along the fissure between the VIII and IX lobule areas were intensely positive for periodic acid-Schiff reaction specific to glycoconjugates, and in this region, calcium depositions were weakly positive for von Kossa's stain. Electron microscopy also revealed that the calcified Purkinje cells possessed numerous electrondense bodies containing inclusions with cystic structures such as vesicles, mitochondria and lysosomes, and these bodies were mainly composed of calcium and phosphorous. These findings suggest abnormal storage of glycoconjugates might be a trigger of Purkinje cell degeneration and serves as a matrix for accumulation of calcium phosphate in the cerebellum of $\mathrm{CC}$ rats.
\end{abstract}

Key words: ataxia, calcification, cerebellum, glycoconjugate, Purkinje cell degeneration 


\section{Introduction}

We found a neurological mutant rat that exhibited a hesitant and wobbling gait with asynergic limb movement and slight tremor, observable after 16-18 days of age [1]. This mutation was clarified as inherited by an autosomal recessive trait. Pathological examination at 3 weeks of age revealed severe degeneration of the Purkinje cells in the cerebellum although the basic layer structure of the cerebellar cortex was not altered. Calcified deposition was recognized by von Kossa's stain in the Purkinje cell somata and dendritic branches. Dotlike deposits were initially observed in the cell somata and calcification appeared to extend to their processes with progression of the disease. In more advanced and severe lesions, calcium deposition developed into many large aggregates in the Purkinje cell and molecular layers, leading to a disruption of the laminar structure. $\mathrm{X}$-ray micrographs showed that calcifications were distributed symmetrically in the whole cerebellar cortex. Glycoconjugates were also observed extensively in the Purkinje cell and molecular layer with the periodic acidSchiff (PAS) reaction. We therefore named this neurological mutant the cerebellar calcification (CC) rat with the gene symbol $c c$. In addition, genetic mapping identified the $c c$ locus as the region near the telomere of the short arm in rat chromosome (Chr) 15, the syntenic region of which is suggested to exist in mouse Chr 14 and human Chr 3 or Chr 10 [2].

In the present study, histopathological examinations were performed in order to investigate the initial process of the Purkinje cell degeneration in postnatal suckling CC rats without clinical signs, which were genotyped by microsatellite markers. In addition, to characterize the calcified Purkinje cells in detail, fine structures of the cells and elemental components in the calcified deposits were examined in fully affected rats by electron microscopy. In the discussion based on the results of these examinations, we suggest that abnormal storage of glycoconjugates might be a trigger of Purkinje cell degeneration and serve as an absorbent for calcium deposition in the cerebellum of CC rats.

\section{Materials and Methods}

\section{Animals}

Sixty-three $\mathrm{CC} \times(\mathrm{CC} \times \mathrm{WKY}) \mathrm{F} 1$ backcross prog- eny were generated as reported previously [2]. Each progeny was genotyped by microsatellite markers at two loci, D15M14Mit222 and D15M14Mit2, located 3.6 $\mathrm{cM}$ distal and $2.7 \mathrm{cM}$ proximal to the $c c$ locus, respectively, through a PCR procedure with genomic DNA prepared from the tail. Rats with CC type markers at both loci were regarded as $c c / c c$ homozygotes and those with $(\mathrm{CC} \times \mathrm{WKY}) \mathrm{F} 1$ type markers were taken as $c c /+$ heterozygotes. These genotyped rats were used for histopathological study at the early postnatal stage. For analyses on the detailed structure and chemical components of the calcified deposits, fully affected CC rats were used at 20 weeks of age. All animals were kept under a controlled temperature $\left(23 \pm 1^{\circ} \mathrm{C}\right)$ with free access to water and rodent chow (CMF, Oriental Yeast Co., Ltd.). The experimental protocol was approved by the Ethics Review Committee for Animal Experimentation of Sankyo Co., Ltd.

\section{Light microscopy}

After collection of the tissue samples for DNA preparation under deep anesthesia with chloral hydrate, rats were sacrificed by transcardiac perfusion with a phosphate buffered solution containing 4\% paraformaldehyde. The cerebella were removed and embedded in paraffin. Mid-saggital sections $6 \mu \mathrm{m}$ thick were stained with hematoxylin and eosin (HE) for general pathological observation, with the periodic acid-Schiff (PAS) procedure to detect glycoconjugates, and with von Kossa's stain to detect the presence of calcium salts. For the sections processed with von Kossa's stain, cell nuclei were counterstained in red with nuclear fast red. Purkinje cells with pyriform somata, an eosinophilic cytoplasm and a large clear ovoid nucleus were regarded as morphologically normal cells and their number was counted in the mid-saggital section stained with HE for each cerebellar lobule.

\section{Electron microscopy}

Rats were sacrificed by transcardiac perfusion of $2.5 \%$ glutalaldehyde in $0.1 \mathrm{M}$ cacodylate buffer $(\mathrm{pH}$ 7.3) under deep anesthesia with chloral hydrate. The cerebella were trimmed into small pieces immediately after surgical removal, stored overnight in the same fixation mixture at $4^{\circ} \mathrm{C}$, and postfixed in $1 \%$ osmium tetroxide for an hour. Then, they were dehydrated through a graded series of ethanol, and embedded in 
Epon 812. Ultrathin sections were stained with uranyl acetate followed by lead citrate and examined with an electron microscope (H-7000, Hitachi Co., Ltd.).

\section{Energy dispersive X-ray spectroscopy}

Specimens for electron microscopy were prepared as described above, and sections were made approximately $100 \mathrm{~nm}$ in thickness and mounted on a copper grid. They were photographed without staining and analyzed with an electron microscope (H-7100, Hitachi Co., Ltd.) equipped with a scanning device and energy dispersive X-ray spectroscopy unit (EMAX-7000, Horiba Co., Ltd.).

\section{Statistics}

Student's $t$-tests were performed on body weight and the number of Purkinje cells to analyze the significance of the differences between homozygous $(c c / c c)$ and heterozygous $(c c /+)$ rats.

\section{Results}

\section{Body growth in the early postnatal stage}

Out of 63 backcross progeny from $\mathrm{CC} \times(\mathrm{CC} \times \mathrm{WKY})$ F1 cross, 30 were genotyped as homozygotes $(c c / c c)$ and the rest were determined as heterozygotes $(c c /+)$. The body weight of the $c c / c c$ rats was already slightly lower at 6 days of age as compared with that of the $c c /+$ rats (Fig. 1). Thereafter, up to 14 days of age, both genotypes showed a similar growth rate and developed no abnormal behavior. However, the growth rate of the $c c / c c$ rats decreased at 14 to 18 days of age and their body weight became about $75 \%$ that of the $c c /+$ rats at 18 days of age. Ataxic behavior characterized by lack of coordination was observed in the $c c / c c$ rats at this age.

\section{Postnatal Purkinje cell degeneration in the cerebellum}

Histologically, the cerebellum of the $c c / c c$ rats was comparable to that of the $c c /+$ rats in size and lobulation throughout the postnatal stage examined. Table 1 shows the number of morphologically normal Purkinje cells in the mid-saggital section stained with HE for each cerebellar lobule. At 6 and 10 days of age, the number of cells in the $c c / c c$ rats was comparable to that of the $c c /+$ rats. However, the number of cells decreased significantly in lobules VI, VII and VIII of the

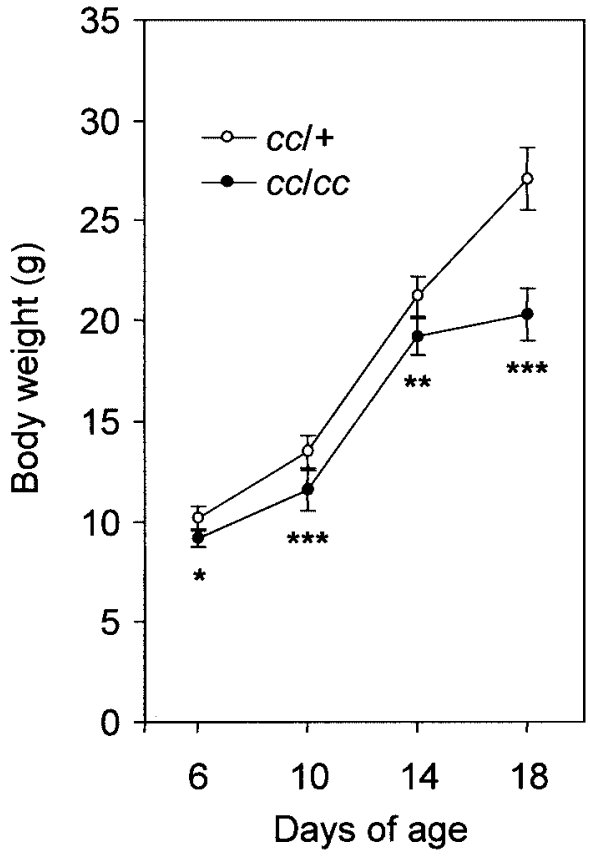

Fig. 1. Body weight of $\mathrm{CC}$ rats in the early postnatal stage. For the number of animals for each plot, see Table 1. All data are shown as means \pm S.D. Statistical significance in the difference between $c c /+$ and $c c / c c$ rats at each age by Student's $t$-test is represented as $* P<0.05, * * P<0.01$ or $* * * P<0.001$.

$c c / c c$ rats at 14 days of age (Table 1 and Fig. 2A, B). And at 18 days of age, $60-70 \%$ of Purkinje cells were degenerated in lobules VII, VIII and IX, and about $40 \%$ were lost in lobule VI. In the other lobules, more than $70 \%$ of Purkinje cells were morphologically normal. In contrast, the number of Purkinje cells remained constant or slightly increased in the $c c /+$ rats. On the other hand, PAS reaction revealed an accumulation of glycoconjugates in the Purkinje cell bodies and their dendrites in the region along the fissure between lobules VIII and IX in 14- and 18-day-old $c c / c c$ rats (Fig. 2C, D). In addition, von Kossa's stain showed slight calcium deposition in the PAS-positive areas in the 18day-old $c c / c c$ rats.

\section{Electron microscopic analysis of mineralized Purkinje cells}

In the cerebellar cortex of the fully affected $\mathrm{CC}$ rats, many dendritic branches of Purkinje cells and their somata were strongly stained by von Kossa's reaction 

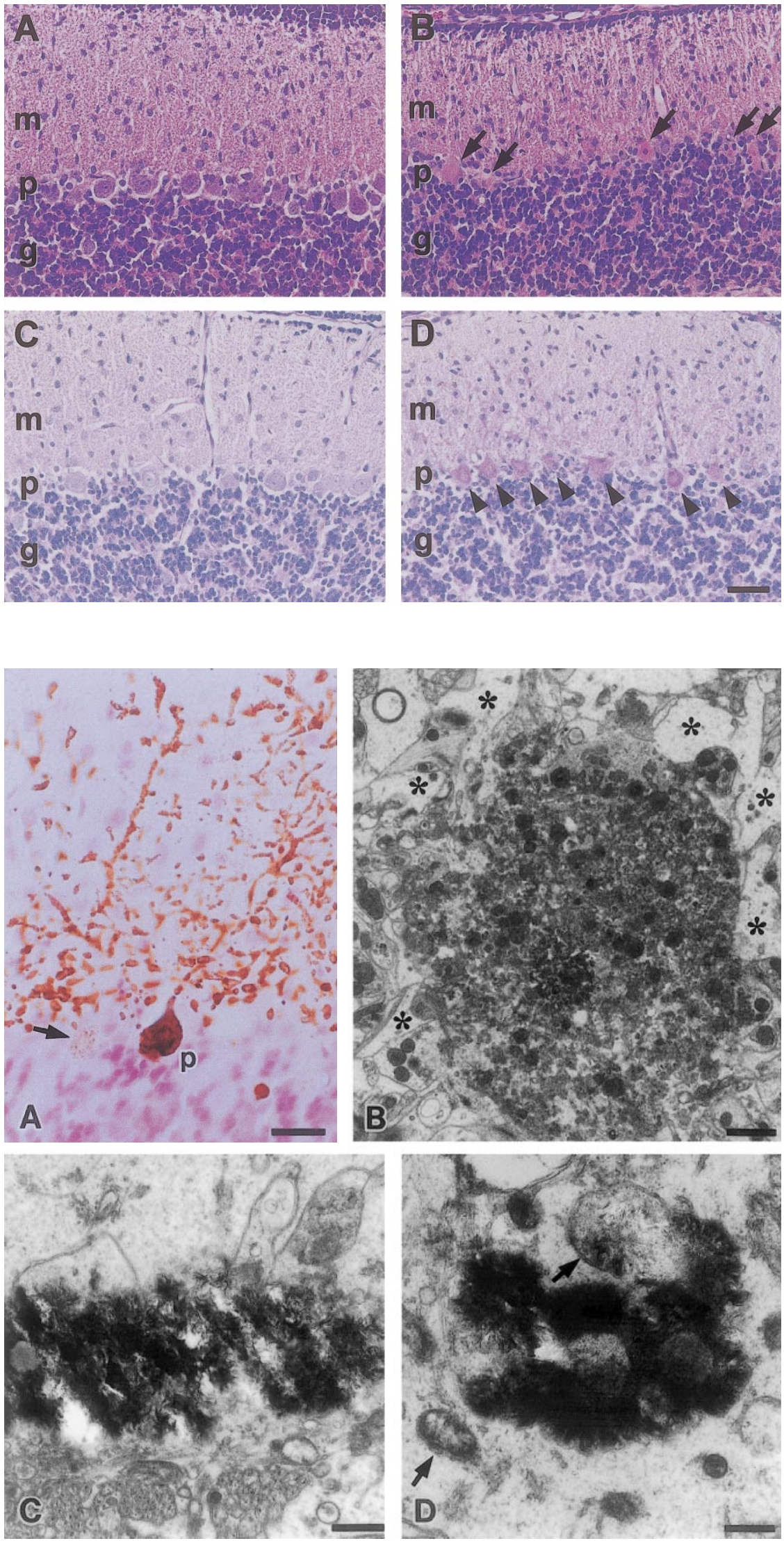

Fig. 2.

Histological changes of the cerebellar Purkinje cells in early postnatal $\mathrm{CC}$ rats. The mid-sagittal sections were stained with hematoxylin and eosin (A, B) and periodic acid-Schiff (PAS) (C, D). In the 14-day-old $c c$ / $c c$ rats $(\mathrm{B})$, loss and degeneration of Purkinje cells (arrows) were observed, but not in the normal $\mathrm{cc} /+$ littermates (A). Some Purkinje cells of the 18-day-old $c c / c c$ rats contained glycoconjugates (arrow heads) stained by PAS reaction (D). No Purkinje cells in the normal $\mathrm{cc} /+$ littermates were PAS-positive (C). M, $\mathrm{p}$ and $\mathrm{g}$ represent the molecular, Purkinje cell and granule layers, respectively. Scale bars represent 50 $\mu \mathrm{m}$.

Fig. 3.

Electron microscopic analysis of the Purkinje cells in $\mathrm{CC}$ rats. (A) Calcified Purkinje cells in $\mathrm{CC}$ rats at 20 weeks of age were positive for von Kossa's stain. In the vicinity of a strongly stained Purkinje cell body (P) and dendrites, dot-like deposits are observed in the degenerating and collapsing Purkinje cell body (arrow). Scale bar represents $20 \mu \mathrm{m}$. (B) Electron micrograph of an initially calcified Purkinje cell at 20 weeks of age. The soma is filled with electron-dense bodies of various sizes and shapes and surrounded by the cytoplasmic processes of astrocytes (asterisks). Scale bar represents $1.5 \mu \mathrm{m}$. (C) Electron micrograph of the molecular layer at 20 weeks of age. Aggregates of needlelike electron-dense bodies (crystals) have penetrated across the cytoplasmic membranes. Scale bar represents $0.7 \mu \mathrm{m}$. (D) High power electron micrograph of the molecular layer at 20 weeks of age. Electrondense bodies have been incorporated into the cystic structures (arrows). Scale bar represents $0.4 \mu \mathrm{m}$. 
Table 1. The number of Purkinje cells in the mid-saggital section of each cerebellar lobule in $c c /+$ and $c c / c c$ rats at early postnatal stage

\begin{tabular}{|c|c|c|c|c|c|c|c|c|c|c|c|c|c|}
\hline \multirow{2}{*}{$\begin{array}{c}\text { Age } \\
\text { (days) }\end{array}$} & \multirow{2}{*}{ Genotype } & \multirow{2}{*}{$\mathrm{N}$} & \multicolumn{11}{|c|}{ No. of Purkinje cells in each cerebellar lobule } \\
\hline & & & I & II & III & IV & V & VI & VII & VIII & IX & $\mathrm{X}$ & Total \\
\hline \multirow{2}{*}{6} & $c c /+$ & 7 & $59 \pm 8$ & $76 \pm 12$ & $86 \pm 12$ & $81 \pm 17$ & $105 \pm 7$ & $203 \pm 18$ & $96 \pm 12$ & $89 \pm 9$ & $77 \pm 8$ & $64 \pm 5$ & $935 \pm 72$ \\
\hline & $c c / c c$ & 5 & $55 \pm 6$ & $76 \pm 13$ & $91 \pm 9$ & $88 \pm 8$ & $98 \pm 3$ & $194 \pm 13$ & $92 \pm 6$ & $83 \pm 13$ & $68 \pm 9$ & $63 \pm 6$ & $908 \pm 45$ \\
\hline \multirow{2}{*}{10} & $c c /+$ & 12 & $56 \pm 10$ & $74 \pm 12$ & $95 \pm 9$ & $86 \pm 16$ & $120 \pm 19$ & $179 \pm 37$ & $91 \pm 11$ & $86 \pm 12$ & $91 \pm 19$ & $84 \pm 11$ & $961 \pm 53$ \\
\hline & $c c / c c$ & 7 & $52 \pm 11$ & $76 \pm 17$ & $100 \pm 15$ & $89 \pm 12$ & $122 \pm 17$ & $170 \pm 34$ & $88 \pm 11$ & $79 \pm 11$ & $102 \pm 9$ & $86 \pm 12$ & $965 \pm 63$ \\
\hline \multirow{2}{*}{14} & $c c /+$ & 8 & $58 \pm 4$ & $82 \pm 13$ & $104 \pm 13$ & $85 \pm 10$ & $118 \pm 17$ & $203 \pm 14$ & $83 \pm 7$ & $97 \pm 14$ & $90 \pm 15$ & $83 \pm 12$ & $1,003 \pm 73$ \\
\hline & $c c / c c$ & 6 & $57 \pm 7$ & $81 \pm 10$ & $105 \pm 12$ & $92 \pm 7$ & $116 \pm 9$ & $167 \pm 15^{\mathrm{c})}$ & $69 \pm 7^{b)}$ & $68 \pm 13^{b)}$ & $81 \pm 10$ & $81 \pm 5$ & $916 \pm 56^{a)}$ \\
\hline \multirow{2}{*}{18} & $c c /+$ & 6 & $60 \pm 5$ & $85 \pm 8$ & $108 \pm 11$ & $92 \pm 7$ & $131 \pm 10$ & $213 \pm 30$ & $97 \pm 11$ & $110 \pm 8$ & $94 \pm 10$ & $98 \pm 15$ & $1,108 \pm 57$ \\
\hline & $c c / c c$ & 12 & $54 \pm 7$ & $72 \pm 9^{a)}$ & $88 \pm 11^{\text {b) }}$ & $80 \pm 13$ & $109 \pm 11^{b)}$ & $124 \pm 20^{\mathrm{c})}$ & $\left.40 \pm 17^{c}\right)$ & $34 \pm 8^{\mathrm{c})}$ & $42 \pm 12^{\mathrm{c})}$ & $71 \pm 10^{\mathrm{c})}$ & $\left.715 \pm 76^{c}\right)$ \\
\hline
\end{tabular}

The number of morphologically normal Purkinje cells was counted in a mid-sagittal section stained with hematoxylin and eosin. All results are shown as mean \pm S.D. Statistical significance in difference between $c c /+$ and $c c / c c$ rats at each age by Student's $t$-test is represented as ${ }^{\text {a) }} P<0.05$, b) $P<0.001$ or $^{\text {c) }} P<0.001$.

(Fig. 3A). Electron microscopic observations revealed that most of the initially calcified somata were filled with electron-dense oval granules and amorphous lowdensity bodies throughout the cytoplasm, and were in contact with many thick cytoplasmic processes of the astrocytes (Fig. 3B). The electron-dense bodies in the calcified dendritic branches were irregular in shape and larger in size compared with those of the somata. They were composed of densely packed needle-shaped crystals and their outlines were brush-shaped (Fig. 3C). Some of the aggregates penetrated out through the cytoplasmic membranes. The electron-dense bodies were also observed inside double membrane cystic structures such as vesicles, mitochondria and lysosomes in both the somata and dendrites (Fig. 3D). The parallel fibers derived from the granule cells in the molecular layer appeared normal. Purkinje cells of the 18-day-old CC rats showed similar pathological changes although accumulated aggregates were sparse.

$X$-ray spectra of electron-dense bodies in Purkinje cells

Figure 4 shows X-ray spectra obtained from representative regions inside and outside the electron-dense bodies in the soma of a Purkinje cell. Significant peaks for both calcium and phosphorous were obtained from spot 1 through spot 4, which were located inside the electrondense body. However, neither peak was conspicuous in the case of spot 5 located outside the dense body.

\section{Discussion}

In the present study, we investigated the initial stage of Purkinje cell degeneration in the cerebellum of $\mathrm{CC}$ rats and examined the fine structures and elemental components in affected cells by electron microscopy.

The basal neuronal network of the cerebellar cortex in rodents is constructed rapidly in the first 3 weeks after birth. The Purkinje cells begin to extend dendritic projections into the molecular layer several days after birth and form synapses with parallel fibers derived from granule cells [3]. In 10-day-old $c c / c c$ rats, the cerebellum was normal in size and lobulation and possessed a lot of morphologically normal Purkinje cells. In addition, immunological staining with calbindin antibody, which is a marker of the Purkinje cell, revealed fertile dendritic trees, and normal synaptogenesis was observed between the dendrites and parallel fibers by electron microscopic analysis (unpublished data). Therefore, we consider that the development of the Purkinje cells of $c c / c c$ rats progresses normally up to 10 days of age.

The growth rate of the $c c / c c$ rats was comparable with that of the $c c /+$ rats during 6-14 days of age, although the $c c / c c$ rats were slightly smaller than the $c c /+$ rats in body weight. This suggests that the $c c$ mutation may be connected with body growth in the fetal and neonatal stages during which the neuronal network of the cerebellum is not yet completed. In contrast, the body weight of the $c c / c c$ rats was lower compared with that of the $c c /+$ rats at 18 days of age, probably reflecting the influence of ataxic behavior. A marked decrease in the number of Purkinje cells was observed in cerebellar lobules VI, VII, VIII and IX after 14 days of age. It is known that the vermis of 

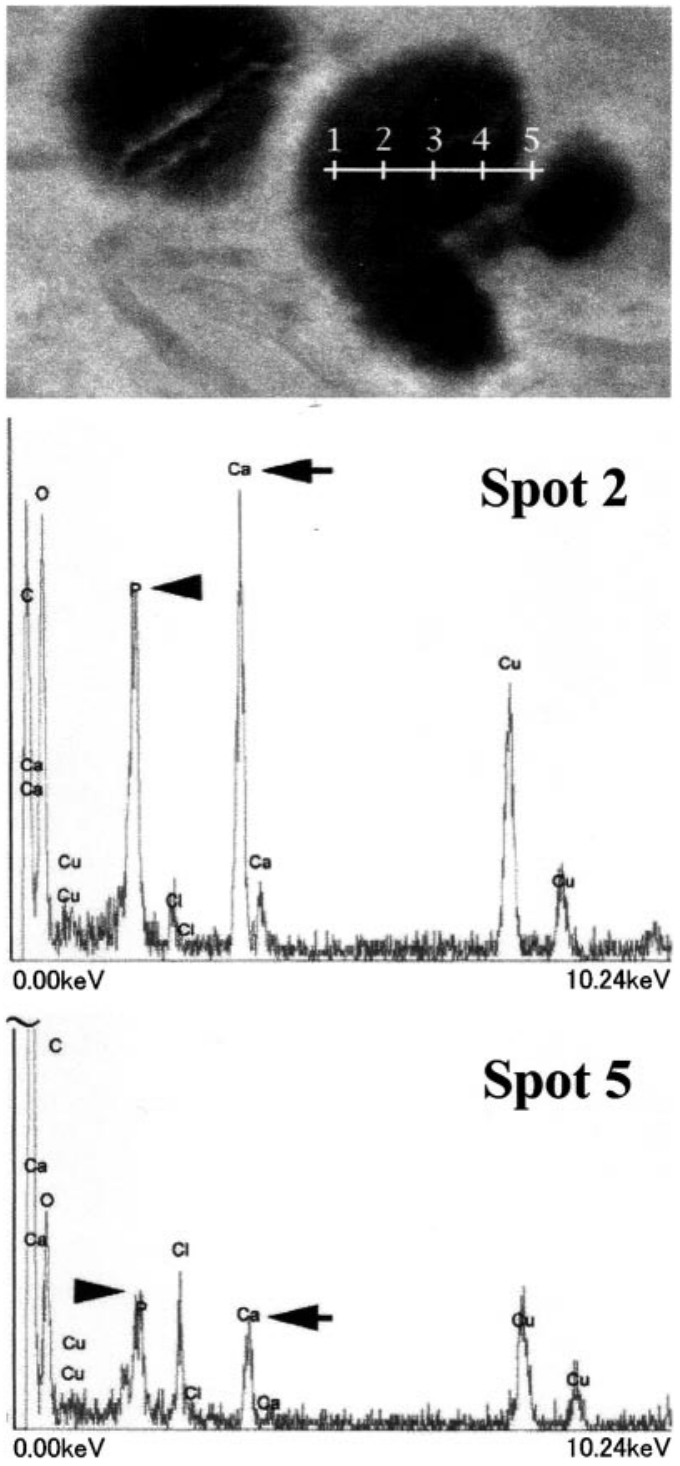

Fig. 4. $X$-ray spectra of an electron-dense body in the Purkinje cell of a CC rat at 20 weeks of age. Xray spectra were obtained from five spots shown in an elec tron micrograph. Spots 1 to 4 are located inside the electron-dense body and spot 5 is located outside the body. Representative X-ray spectra of spots 2 and 5 are shown. Atomic symbols are given for each peak. Spot 2 contained higher peaks of $\mathrm{Ca}$ (arrow) and $\mathrm{P}$ (arrow head) compared with spot 5 . Peaks for $\mathrm{Cu}$ and $\mathrm{Cl}$ are due to contamination from grid metal and Epon resin, respectively.

these lobules plays a role in regulating the muscle tone of the limbs [4]. Thus, the temporal and spatial progress of the Purkinje cell degeneration in CC rats seems to be coincident with the appearance of ataxia and its char- acteristics, i.e. asynergic movement of the limbs.

Spontaneous mutation arising from severe Purkinje cell loss has been reported in shaker rats, nervous mice and Purkinje cell degeneration $(p c d)$ mice. Shaker rats exhibit adult-onset loss of Purkinje cells particularly in the anterior lobules of the cerebellum [5]. In nervous mice, Purkinje cell loss occurs rapidly after birth and more extensively in the hemisphere than in the vermis [6]. In $p c d$ mice, Purkinje cell loss develops throughout the cerebellum postnatally [7]. In contrast, Purkinje cell loss of CC rats occurs progressively from the posterior lobules postnatally and seems to take place more extensively in the vermis than in the hemisphere judging from the distribution of calcified deposits [1]. Thus, there are some differences in the temporal and spatial sequences of Purkinje cell loss between these mutants and $\mathrm{CC}$ rats. In addition, different pathological features also exist in other cells: most of the olivary neurons degenerate in shaker rats; the photoreceptor cells of the retina is affected in nervous mice; and degeneration of photoreceptor cells of the retina and mitral cells of the olfactory bulb as well as sperm loss occur in pcd mice [5-7]. However, in CC rats, no pathological changes have been detected except for Purkinje cell degeneration and disruption of molecular layers by calcification, although suppression of body growth and very low fertility of males are observed. Therefore, this evidence suggests that Purkinje cell degeneration in $\mathrm{CC}$ rats may be caused by a different pathogenic process from that in the other three mutants.

In the cerebellum of $c c / c c$ rats, PAS-positive substances accumulated in the somata and dendrites of the Purkinje cells during the growth period when synaptogenesis developed rapidly in the Purkinje cells. The accumulating substances are thought to be glycoconjugates containing glycoproteins, proteoglycans and glycolipids since glycogen is removed by diastase digestion [1]. Many glycoconjugates play a critical role in the morphogenesis, synaptogenesis and maintenance of the Purkinje cells [8-11], but pathogenic storage of glycoconjugates often results in neurodegeneration known as a lysosomal disorder, which is caused by genetic defects of hydrolytic enzymes and their related proteins [12]. Gene-deficient mice on hexosaminidase $\mathrm{B}$ or $\mathrm{G}_{\mathrm{M} 2}$ activator protein, models of $\mathrm{G}_{\mathrm{M} 2}$ gangliosidosis, display significant storage of $\mathrm{G}_{\mathrm{M} 2}$ gangliosides with sialic acid in neuronal 
cells including the Purkinje cells in restricted regions of the brain, resulting in a defect in balance and motor coordination [13, 14]. However, hexosaminidase Adeficient mice, which are another model of $\mathrm{G}_{\mathrm{M} 2}$ gangliosidosis, exhibit only minimal pathology in the cerebellum and are asymptomatic [15]. Thus, glycoconjugate accumulation seems to cause dysfunction of the cerebellar neuronal cells including the Purkinje cells and lack of coordination. In the present study, histological examination showed that PAS-positive Purkinje cells of $\mathrm{CC}$ rats in the postnatal and adult stages had high affinity to a lectin (MAL2) recognizing glycoconjugates with sialic acid (unpublished data). This evidence suggests that pathologic storage of glycoconjugates is a trigger of the Purkinje cell degeneration in $\mathrm{CC}$ rats. However, further investigation is needed to explain the pathological changes characteristic to the cerebellum in $\mathrm{CC}$ rats.

Electron microscopy revealed that the Purkinje cells of $\mathrm{CC}$ rats possess vesicles and mitochondria with coalescing electron-dense substances as well as lysosome-like dense bodies. X-ray micro-analysis also clarified that these electron-dense bodies contained calcium and phosphorous. These findings suggest that the calcified deposits penetrated into the cystic structures with glycoconjugates. In some examples of intracranial calcification in humans and rodents, affected sites have been shown to contain glycoconjugates that are positive to the PAS reaction [16-19]. It is also known that proteoglycans are involved in the formation and development of calcified tissues such as bones and teeth [20]. Taken together, it seems likely that the glycoconjugates serve as an absorption matrix for incorporating calcium phosphate in the cerebellum of CC rats. The neurological degeneration of $\mathrm{CC}$ rats is inherited as an autosomal recessive trait $[1,2]$. Therefore, $\mathrm{CC}$ rats may provide a key for clarifying the deranged pathway that interferes with the construction of the central nervous system due to genetic defects.

\section{References}

1. Ando, Y., Ichihara, N., Takeshita, S., Nagata, M., Kimura, T., Tanase, H., and Kikuchi, T. 1999. A new neurological mutant rat with symmetrical calcification of Purkinje cells in cerebellum. Proc. Soc. Exp. Biol. Med. 221: 361-368.

2. Ando, Y., Takeshita, S., Fujimoto, K., Shimizu, M., Nagata, M., Kikuchi, T., and Wakasugi, N. 2001. Genetic mapping of a neurological mutation cerebellar calcification in the rat. Mamm. Genome 12: 80-82.

3. Altman, J. and Bayer, S.A. 1996. Development of The Cerebellar System, CRC Press, Florida.

4. Breazile, J.E. 1977. Cerebellum. pp. 617-619. In: Duke's Physiology of Domestic Animals, 9th ed. (Swenson M. J. ed.), Cornell University Press Ltd., London.

5. La Regina, M.C., Yates-Siilata, K., Woods, L., and Tolbert, D. 1991. Preliminary characterization of hereditary cerebellar ataxia in rats. Lab. Anim. Sci. 42: 19-26.

6. Green, M.C. 1989. Catalog of mutant genes and polymorphic loci. pp. 262-263. In: Genetic Variation and Strains of the Laboratory Mouse (Lyon, M.F. and Searles, A.G., eds.), Oxford University Press, New York.

7. Mullen, R.J., Eicher, E.M., and Sidman, R.L. 1976. Purkinje cell degeneration, a new neurological mutation in the mouse. Proc. Natl. Acad. Sci. USA 73: 208-212.

8. Furuya, S., Hashikawa, T., and Hirabayashi, Y. 1996. Restricted occurrence of an unusual ganglioside GD1 alpha in rat brain and its possible involvement in dendritic growth of cerebellar Purkinje neurons. J. Neurosci. Res. 44: 7383.

9. Tanaka M., Maeda, N., Noda, M., and Marunouchi, T. 2003. A chondroitin sulfate proteoglycan PTPzeta / RPTPbeta regulates the morphogenesis of Purkinje cell dendrites in the developing cerebellum. J. Neurosci. 23: 2804-2814.

10. Aono, S., Keino, H., Ono, T., Yasuda, Y., Tokita, Y., Matsui, F., Taniguchi, M., Sonta, S., and Oohira, A. 2000. Genomic organization and expression pattern of mouse neuroglycan C in the cerebellar development. J. Biol. Chem. 275: 337-342.

11. Chou, D.K., Suzuki, Y., and Jungalwala, F.B. 1996. Expression of neolactoglycolipids: sialosyl-, disialosyl-, Oacetyldisialosyl- and fucosyl- derivatives of neolactotetraosyl ceramide and neolactohexaosyl ceramide in the developing cerebral cortex and cerebellum. Glycoconj. J. 13: 295-305.

12. Lyon, G., Adams, R., and Kolodny, E. 1996. Neurology of Hereditary Metabolic Disease of Children, $2^{\text {nd }}$ ed., McGrawHill, New York.

13. Taniike, M., Yamanaka, S., Proia, R.L., Langaman, C., Bone-Turrentine, T., and Suzuki, K. 1995. Neuropathology of mice with targeted disruption of Hexa gene, a model of Tay-Sachs disease. Acta Neuropathol. 89: 296-304.

14. Liu, Y., Hoffmann, A., Grinberg, A., Westphal, H., McDonald, M.P., Miller, K.M., Crawley, J.N., Sandhoff, K., Suzuki, K., and Proia, R.L. 1997. Mouse model of GM2 activator deficiency manifests cerebellar pathology and motor impairment. Proc. Natl. Acad. Sci. USA 94: 8138-8143.

15. Sango, K., Yamanaka, S., Hoffmann, A., Okuda, Y., Grinberg, A., Westphal, H., McDonald, M.P., Crawley, J.N., Sandhoff, K., Suzuki, K., and Proia, R.L. 1995. Mouse models of Tay-Sachs and Sandhoff diseases differ in neurologic phenotype and ganglioside metabolism. Nat Genet. 11: 170-176. 
16. Kurup, R.K. and Kurup, P.A. 2002. Hypothalamic digoxin related membrane $\mathrm{Na}+\mathrm{K}+$ ATPase inhibition and familial basal ganglia calcification. Neurosci. Res. 42: 35-44.

17. Fujita, D., Terada, S., Ishizu, H., Yokota, O., Nakashima, H., Ishihara, T., and Kuroda, S. 2003. Immunohistochemical examination on intracranial calcification in neurodegenerative diseases. Acta Neuropathol. 105: 259264.

18. Kobayashi, S., Yamadori, I., Miki, H., and Ohmori, M. 1987. Idiopathic nonarteriosclerotic cerebral calcification (Fahr's disease): an electron microscopic study. Acta
Neuropathol. 73: 62-66.

19. Yanai, T., Masegi, T., Ueda, K., Manabe, J., Teranishi, M., Takaoka, M., Matsunuma, N., Fukuda, K., and Goto, N. 1993. Spontaneous globoid mineralization in the cerebellum of rats. J. Comp. Pathol. 109: 447-451.

20. Hoshi, K., Ejiri, S., and Ozawa, H. 2001. Localizational alterations of calcium, phosphorus, and calcification-related organics such as proteoglycans and alkaline phosphatase during bone calcification. J. Bone Miner. Res. 16: 289298. 